\title{
Arthroscopic treatment of anteromedial ankle impingement
}

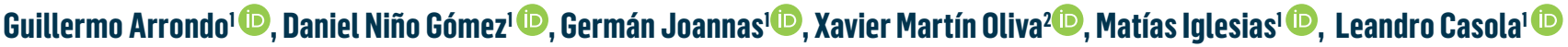 \\ 1. Sanatorio Dupuytren, Autonomous City of Buenos Aires, Buenos Aires, Argentina. \\ 2. Clinica Remei, Barcelona, Spain.
}

\begin{abstract}
Objective: Impingement syndromes are recognized as an important cause of chronic ankle pain, which results from the entrapment of an inflamed soft-tissue component between the osteophytes. The predominant site of occurrence is the anterolateral aspect of the ankle for soft-tissue impingement, and anteromedial aspect for bony impingement. Symptoms related to the physical impact of bone or soft-tissue pain often result in limited ankle range of motion.
\end{abstract}

Methods: We conducted a retrospective study of 34 patients (34 ankles) with anteromedial bony impingement. All patients underwent arthroscopy, with a mean follow-up of 34 months.

Results: All osteophytes were removed, and the ankle range of motion improved. The AOFAS score improved from 73 preoperatively to 95 postoperatively.

Conclusion: The arthroscopic removal of the anteromedial osteophytes of the ankle had excellent functional results. It is an effective procedure that allows rapid patient recovery.

Level of Evidence IV; Therapeutic Studies; Case Series.

Keywords: Ankle injuries/complications; Exostoses/surgery; Ankle injuries; osteophytes; Arthroscopy; Sports.

\section{Introduction}

Impingement syndromes, recognized as a significant cause of chronic ankle pain, have been described in the anterior, posterior, anterolateral, and anteromedial region of the ankle. While these conditions are relatively rare, their diagnosis is considered important as they can lead to chronic ankle pain. Murawski and Kennedy explain in their article that the pain is secondary to the entrapment of an inflamed soft tissue component between the osteophytes ${ }^{(1)}$. Moreover, the predominant site of occurrence is the anterolateral aspect of the ankle for soft-tissue impingement, and anteromedial aspect for bony impingement ${ }^{(2)}$.

In 1943, Morris called this impingement "athlete's ankle". Later, in 1950, McMurray preferred to call it "footballer's ankle" and published good results in athletes after surgical resection. Thereafter, the term "anterior ankle impingement syndrome" has been widely cited. Nowadays, this condition is recognized as 2 different entities, anteromedial impingement syndrome and anterolateral impingement syndrome ${ }^{(1)}$.
Symptoms related to the physical impact of bone or soft-tissue pain often result in limited ankle range of motion. Massada found that as many as $60 \%$ of professional soccer players have osteophytes located anteriorly within the ankle joint ${ }^{(3)}$.

It is a condition that almost exclusively affects athletes as they routinely submit the talocrural joint to repetitive dorsiflexion movements, although there are some cases of patients with no sports activities but who perform repetitive dorsiflexion movements, such as people who work in a squat position or who frequently go up and down stairs. As Manoli mentions in his article, it can also appear after nonsporting injuries, especially fractures about the ankle and foot. Therefore, there could be an association with a subtle cavus foot and ankle instability ${ }^{(4)}$.

McMurray hypothesized 3 primary theories, since he thought that there was no clear explanation for the cause of anteromedial impingement (AMI). First, he hypothesized that the talotibial osteophytes were formed by repetitive capsular traction during kicking movements. This has since become
Study performed at the Institution Dupuytren, Autonomous City of Buenos Aires; Buenos Aires, Argentina.

Correspondence: Matías Iglesias. 1555 Haedo St., Vicente López, Buenos Aires, Argentina, Zip Code: 1605. E-mail: mfiglesias6@gmail.com

Conflicts of interest: none. Source of funding: none. Date received: September 17, 2020. Date accepted: October 31, 2020. Online: December 21, 2020

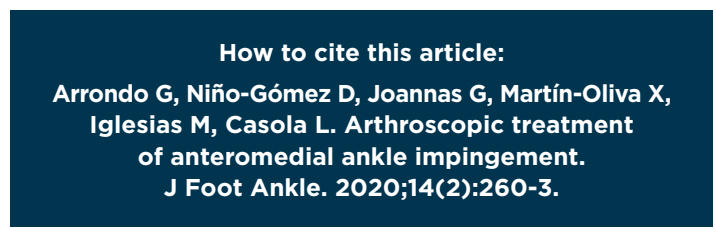


known as the "traction spurs" theory. The second theory relates to direct and recurrent microtrauma to the joint capsule, and he believes that the impact forces of a soccer ball are of a great enough magnitude to incur damage to the anatomy of the anterior ankle region ${ }^{(5)}$. Spur formation and symptoms of AMI can also be a result of repetitive dorsiflexion of the ankle joint; this may be particularly relevant in dancers ${ }^{(1)}$. Open or arthroscopic surgery are a treatment option in cases in which the medial impingement lesion is sufficiently symptomatic.

The aim of the present study was to retrospectively report the outcomes of our patients with anteromedial ankle impingement operated on by arthroscopy.

\section{Methods}

This study was approved by the Institutional Review Board. We conducted a retrospective study of 34 patients (34 ankles) with anteromedial bony impingement; patients with only soft-tissue impingement were excluded. All patients underwent arthroscopy between January 2014 and July 2019, with a mean follow-up of 34 months (minimum, 24 months; maximum, 40 months). Mean patient age was 32.1 years (minimum, 22 years; maximum, 42 years); 30 were men and 4 were women. Seven patients were high-performance athletes, 24 participated in amateur sports, and 3 patients reported low physical activity. Clinically, all patients had pain at the medial ankle joint and limited dorsiflexion.

For all patients, radiographs of the ankle (anteroposterior, lateral, and $45^{\circ}$ oblique) were taken and graded according to the van Dijk scale (Figure 1). The obtained images revealed the presence of osteophytes at the tibial and talar levels in all patients.

Initial treatment was conservative and consisted of administration of nonsteroidal anti-inflammatory drugs (NSAIDs) and physical therapy, which lasted for an average of 4.9 months (minimum, 2 months; maximum, 12 months). Surgery was proposed when there was persistence of symptoms despite conservative treatment. Surgical treatment consisted of arthroscopy of the ankle and removal of the osteophytes shown on the diagnostic radiographs.

Arthroscopy was performed through anteromedial and anterolateral portals with the patient under general anesthesia or spinal block. Prophylactic antibiotic therapy was administered, and a tourniquet was applied to the lower limb in all patients. The joint was visualized and debrided through the arthroscope using associated instruments (Shaver). Presence of exostoses of the talus and tibia was confirmed (Figures 2 and 3).

Spurs were removed with an osteotome or a burr-type device. Fluoroscopic support was performed (Figures 4 and 5).

\section{Results}

Tibial and talar osteophytes were surgically resected in all patients. Radiographs were taken postoperatively to control for exostoses (Figure 6).
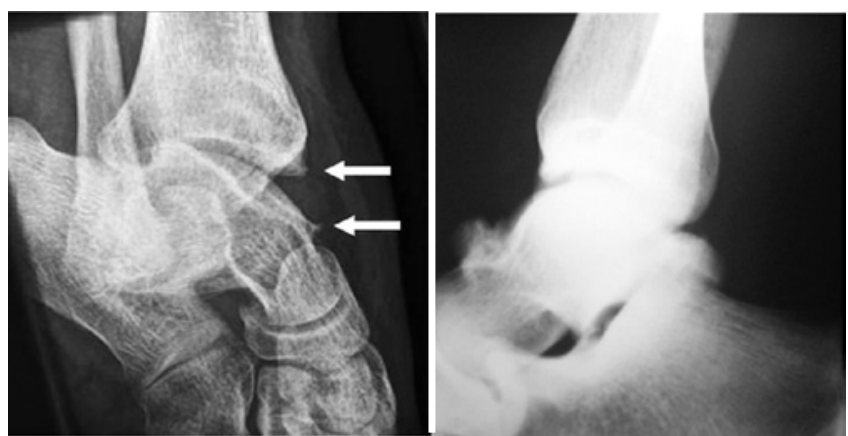

Figure 1. Oblique and lateral radiographs of the ankle. Tibial and talar osteophytes

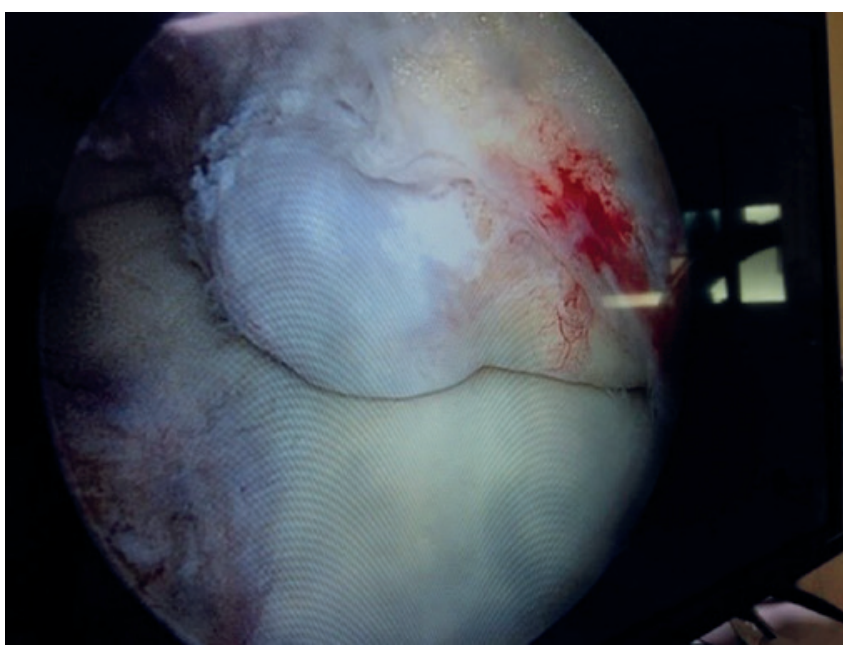

Figure 2. Arthroscopy. Tibial osteophyte.

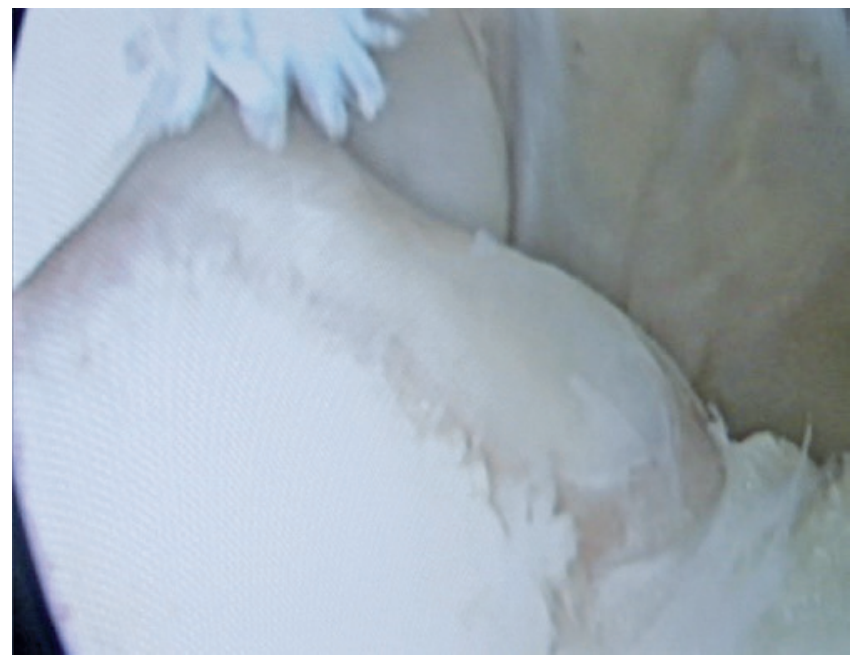

Figure 3. Arthroscopy. Talar osteophyte. 


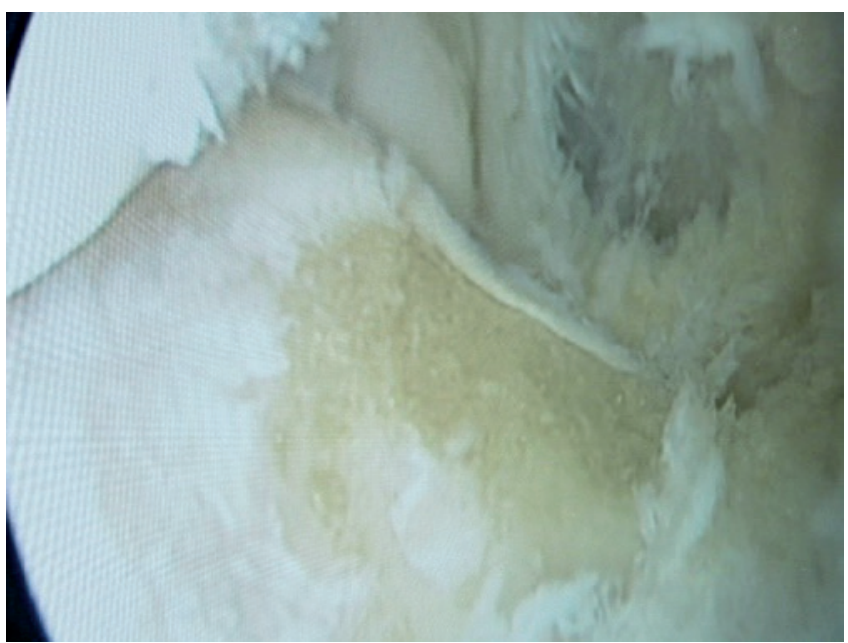

Figure 4. Removal of talar osteophyte.

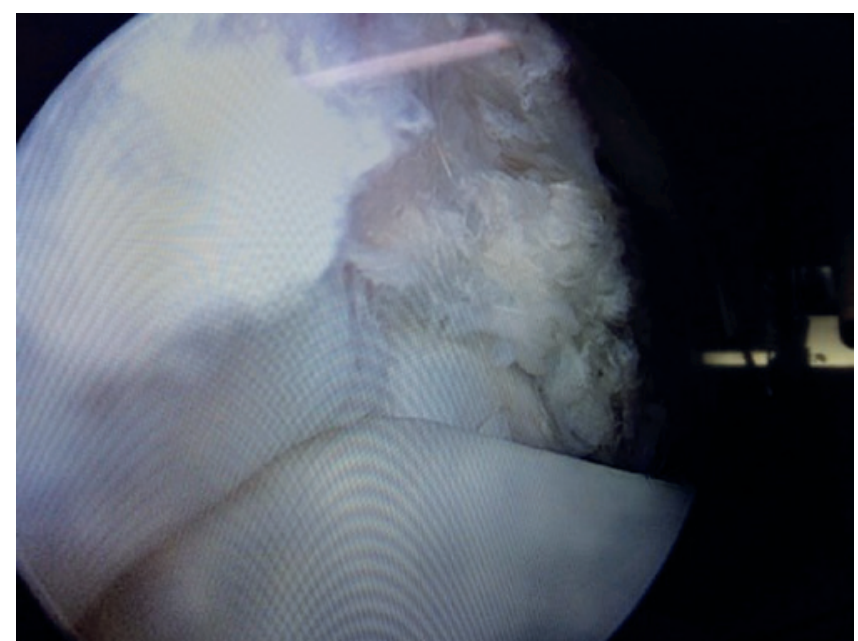

Figure 5. Removal of tibial osteophyte
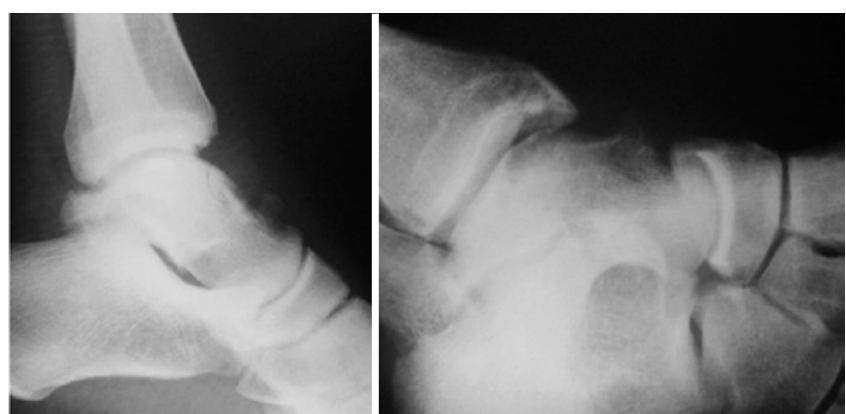

Figure 6. Postoperative lateral and oblique radiographs of the ankle.
Patients started weight-bearing on postoperative day 2. Sutures were removed on day 10. Rehabilitation was performed for 2 months. All patients returned to their daily activities at a mean of 3.8 weeks after surgery. Athletes returned to sport-specific training at a mean of 2.1 months after surgery. The American Orthopaedic Foot and Ankle Society (AOFAS) score improved from 73 preoperatively to 95 postoperatively. All patients had relief of symptoms, were satisfied with the surgical procedure and were willing to recommend it to others. No complications were reported.

\section{Discussion}

Anteromedial ankle impingement is a frequently misdiagnosed pathology that affects athletes and, consequently, their quality of play. The diagnosis is purely clinical, and pain of the ankle joint line is often present along with limited dorsiflexion. Because forced hyperdorsiflexion may produce pain, diagnostic confirmation is needed ${ }^{(6)}$.

In 2000 , Scranton et al. found that $57 \%$ of patients with chronic instability have impingement spurs, against $17 \%$ of 200 randomly selected individuals. They hypothesized that ankle instability is associated with arthritic changes; therefore, impingement spurs occur as a consequence of instability and are the expression of joint degeneration, thereby differing from localized spurs in case of local microtrauma. In fact, chronic ankle instability is associated with osteophytic formation in cases with medial and lateral ankle compartment ${ }^{(7)}$.

The first complementary study for the imaging diagnosis of AMI should be a routine weight-bearing radiograph in anteroposterior and lateral directions. An oblique AMI radiogra$\mathrm{ph}$ is recommended ${ }^{(6)}$. The anterolateral aspect of the tibia is the most prominent aspect on standard lateral radiographs, thereby appreciating anterolateral osteophytes. On the other hand, anteromedial osteophytes are often misdiagnosed on standard lateral projections ${ }^{(1)}$.

Tol and van Dijk cited an interesting cadaver study describing that anteromedial tibial osteophytes up to $7.3 \mathrm{~mm}$ in size and originating from the anteromedial border could be undetected on a standard lateral radiograph because of the prominent aspect of the anterolateral border of the distal tibia $^{(8)}$. As a complement, the sensitivity of lateral radiographs for detecting anterior tibial and talar osteophytes was $40 \%$ and $32 \%$, respectively (specificity $70 \%$ and $82 \%$ ). Also, when the lateral radiograph was combined with an oblique AMI radiograph (craniocaudal radiograph with 30 external rotation of the leg), sensitivity increased to $85 \%$ and $73 \%$, respectively. This increase was due to the high sensitivity of the oblique AMI radiographs for detecting anteromedial osteophytes (93\% for tibial and $67 \%$ for talar osteophytes) ${ }^{(8)}$.

Another complementary study is magnetic resonance imaging (MRI). MRI is useful to detect injuries in the anterior deltoid thickening, synovitis, and ossifications. In the coronal view, one can identify injury to the anterior tibiotalar band of the deltoid, typically seen as ligament thickening and edema. Therefore, with MRI, one can make a differential diagnosis 
with soft-tissue diagnoses, including osteochondral lesions, loose bodies, and stress fractures ${ }^{(6)}$.

Van Dijk et al. were the first to differ between the affected sites in 62 arthroscopic procedures for treatment of anterior ankle impingement ${ }^{(9)}$. They compared AMI resection vs anterolateral impingement (ALI) resection and found that the former was statistically superior at 4 months, 1 year, and 2 years postoperatively. Two years after surgery, $66 \%$ of ALI patients and $87 \%$ of AMI patients had a good to excellent result. Visual analog scale (VAS) results also showed improvement in pain postoperatively. At 2 years, VAS scores in the AMI group had decreased from 6.9 preoperatively to 4.3 postoperatively ${ }^{(6)}$.

Murawski and Kennedy ${ }^{(1)}$ demonstrated the results of the arthroscopic treatment of 41 patients with AMI, with a mean patient age of 31.12 years; 34 patients (83\%) were competing at some level of athletic sport. At a minimum of 2 years of follow-up, 93\% were satisfied with the procedure. The results of both AOFAS (62.83 to 91.17) and Short Form-36 version 2 (61.54 to 92.21) improved significantly, and all but 1 patient returned to their pre-injury level of sporting activity. Ten patients also had a concomitant lateral ligament reconstruction or arthroscopic bone marrow stimulation of an osteochondral lesion. The possibility to return to play in the athletic patients undergoing only AMI resection was 7 weeks. The difference was observed in the patients who had concomitant lateral ligament reconstruction, as they returned to sport at an average of 15 weeks, and in those who underwent concomitant bone marrow stimulation, as they returned to sports at an average of 14 weeks. Patients who were not involved in sports
(17\%) returned to daily activities without pain at an average of 9 weeks after AMI resection ${ }^{(6)}$. No high dorsiflexion range of motion was observed in the ankle; it was slightly limited in all cases before surgery by a mean of $3.2^{\circ}$ (range $2-5^{\circ}$ ) compared to the contralateral side, as explained by the authors. Postoperative results showed a normal range of motion $\left(<2^{\circ}\right.$ of the contralateral side) in all patients. In fact, complications occurred in 3 cases after arthroscopy, accounting for $7 \%$ of the overall cohort. A single patient had neurapraxia of the superficial peroneal nerve, which resolved 6 weeks postoperatively. Another patient developed arthrofibrosis after surgery requiring further manipulation under anesthesia and an injection of triamcinolone. Only 1 patient developed complex regional pain syndrome.

In our patients, all impingement was resected. All patients returned to their daily activities at 3.8 weeks, with a better ankle range of motion. The AOFAS score improved from 73 to 95 . All symptoms were relieved, and all patients were satisfied with the treatment. There were no complications.

\section{Conclusion}

The arthroscopic removal of the anteromedial osteophytes of the ankle produces excellent functional results. It is an effective procedure that allows rapid patient recovery, including early return to sports activities with minimal morbidity associated with the procedure, compared to arthrotomy. In athletes who participate in jumping or kicking sports, the impingement may require removal.

Authors' contributions: Each author contributed individually and significantly to the development of this article: AG *(https://orcid.org/OOOO-OOO34767-5489) conceived and planned the activities that led to the study, interpreted the results of the study and approved the final version; DNG *(https:// orcid.org/0000-0002-7870-5882) conceived and planned the activities that led to the study, interpreted the results of the study and approved the final version; GJ *(https://orcid.org/0000-0001-9998-190X) bibliographic review, interpreted the results of the study, clinical evaluation of the patients and performed the surgeries; XMO *(https://orcid.org/0000-0003-2231-0678) conceived and planned the activities that led to the study, interpreted the results of the study and approved the final version; MI *(https://orcid.org/0000-0002-6336-6080) bibliographic review and approved the final version; LC *(https://orcid.org/0000-0003-1187-0864) bibliographic review and clinical evaluation of the patients. *ORCID (Open Researcher and Contributor ID) (iD).

\section{References}

1. Murawski CD, Kennedy JG. Anteromedial impingement in the ankle joint: outcomes following arthroscopy. Am J Sports Med. 2010;38(10):2017-24.

2. Zwiers R, Wiegerinck JI, Murawski CD, Fraser EJ, Kennedy JG, van Dijk CN. Arthroscopic Treatment for Anterior Ankle Impingement: A Systematic Review of the Current Literature. Arthroscopy. 2015;31(8):1585-96.

3. Massada JL. Ankle overuse injuries in soccer players. Morphological adaptation of the talus in the anterior impingement. J Sports Med Phys Fitness. 1991;31(3):447-51.

4. Manoli A 2nd. Medial impingement of the ankle in athletes. Sports Health. 2010;2(6):495-502.
5. Tol JL, Slim E, van Soest AJ, van Dijk CN. The relationship of the kicking action in soccer and anterior ankle impingement syndrome. A biomechanical analysis. Am J Sports Med. 2002;30(1):45-50.

6. Ross KA, Murawski CD, Smyth NA, Zwiers R, Wiegerinck JI, van Bergen CJ, et al. Current concepts review: Arthroscopic treatment of anterior ankle impingement. Foot Ankle Surg. 2017;23(1):1-8.

7. Parma A, Buda R, Vannini F, Ruffilli A, Cavallo M, Ferruzzi A, et al. Arthroscopic treatment of ankle anterior bony impingement: the long-term clinical outcome. Foot Ankle Int. 2014;35(2):148-55.

8. Tol JL, van Dijk CN. Anterior ankle impingement. Foot Ankle Clin. 2006;11(2):297-310.

9. van Dijk CN, Tol JL, Verheyen CC. A prospective study of prognostic factors concerning the outcome of arthroscopic surgery for anterior ankle impingement. Am J Sports Med. 1997;25(6):737-45. 